\title{
Experiments with Granular Material Motion for Extraterrestrial Applications
}

\author{
Nathaniel S. Helminiak ${ }^{1}$ \\ Department of Mechanical Engineering \\ Marquette University, Milwaukee
}

\begin{abstract}
In this study, a horizontal drum tumbler, filled at variable depths with spherical media, was rotated at constant speeds. An exposed monoplane layer of aggregate was photographed with a high-speed camera, in order to perform a particle tracking velocimetry (PTV) algorithm followed by image registering. The algorithms extracted both the translational and rotational velocities. To our knowledge, this is the first reported experimental measurement of rotational velocities in a rotary drum tumbler with granular media. The objective of this study, companioned with David Helminiak's "Simulations with Granular Material Motion for Extraterrestrial Applications", sought to measure the induced rotational velocity of individual grains and characterize the distribution of energy, both translational and rotational. The study not only proposes an addition to the existing idea of force chains, namely rotational "Bro-chains", but also suggests that within industry, mass finishing can benefit from complete energy characterization.
\end{abstract}

\section{Background}

Granular flow and related mechanics are uniquely fascinating as they combine mechanics of both fluids and solids. While granular materials tend to conform to their surroundings, they fill space in a much less efficient manner than would their liquid counterparts. Similarly, granular materials behave like solids with regard to packing efficiencies and resistance to shear. In any case, granular material interactions are among the most prevalent phenomenon on earth and in our universe. Through the characterization of these flows, we can better model such everyday processes as mass finishing to such extraordinary ideas as describing the makeup of a meteor strike on Mars.

In industry, granular flow fields induced in a rotating tumbler have a variety of applications, including mixing, segregation processes, as well as mass finishing, such as deburring and surface hardening. A common industrial deburring technique involves immersing the part in a drum with an abrasive aggregate. The drum is then agitated to induce an abrasive action. Burr removal is accomplished by relative velocity between the part and aggregate in contact with the part, as well as the relative rotation between individual aggregate and the part (T. Deng; 2004). In the study of granular flow in mass finishing, the most relevant results of the vibratory motion are those that lead to the grinding and polishing of the chosen media.

Within rotary mass finishing, there exist two main modes of material removal: impact and abrasive rotary friction. Impact occurs when outermost particles are flung upwards, free of the surrounding particles before following a projectile motion path back onto the heap of other aggregates. Impact mechanics of the two methods has had much investigative research both experimental and simulated with emphasis on deformation and mechanics (Warr, S; 1994). The second mode by which material is ground is through erosive wear of the aggregate surfaces stemming from individual particle translations and rotations as they move up and around the cylinder. Improvements in deburring processes can be accomplished by better understanding the relationship between the drum and the induced granular flow.

\footnotetext{
${ }^{1}$ Wisconsin Space Grant Consortium, University of Wisconsin-Green Bay Advisors: Dr. John Borg, P.E., Dr. Vikram Cariapa, P.E
} 
When working in three-dimensional space, problems, especially in high particle density situations, often occur with particles overlapping and obscuring each other leading to lost identities. While some studies suggest that by using colored, flagged, or tracer particles, more information might be gleaned from a single run of particle within a three-dimensional field (Bendicks C; 2011), it still cannot with high repeatability track all particles. Furthermore, PTV or Particle Tracking Velocimetry developed for three-dimensional tracking requires utilizing multiple cameras (Homeniuk, Darren; 2010). Our study, having one camera, focused on two-dimensional flow.

The 2D flow field can be characterized in a variety of ways. A common non-dimensional characterization is the Froude number, in this case defined as

$$
F_{O}=\frac{{ }^{2} D}{g}
$$

where $\omega$ is the rotational rate of the drum, $\mathrm{D}$ is the drum diameter, and $\mathrm{g}$ is gravity $\left(9.81 \frac{\mathrm{m}}{\mathrm{s}^{\mathrm{s}}}\right)$. The Froude number represents the ratio of rotational force to gravitational force or rotational energy to potential energy. When characterized in this way, distinct flow regimes describing the translational velocity field emerge (see Figure 1-1). The exact location of transition of modes (highlighted in Figure 1-2) can change as the ratio of the drum diameter and the granular media vary. Thus, Froude number alone is not sufficient to describe mode of operation.
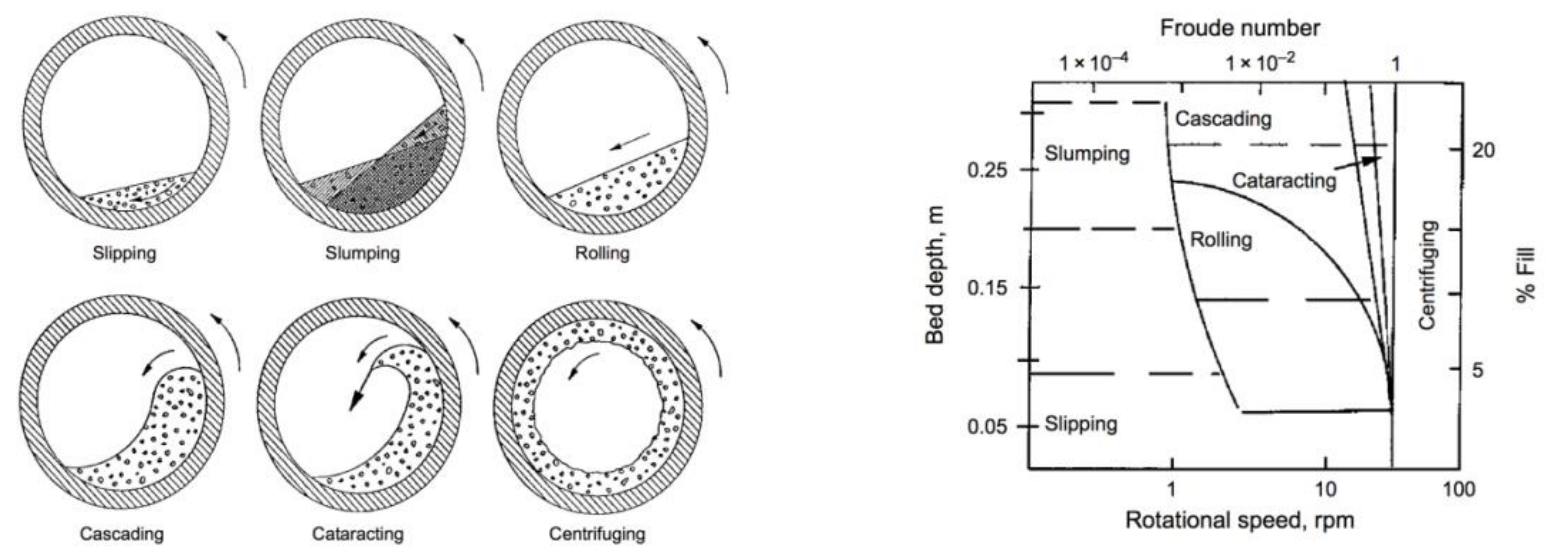

Figures 1-1 and 1-2. Characterization of granular flow in a horizontal rotary tumbler, the results collected by past studies (Valle, Romero; 2012)): the figure on the left displays the six characteristic flow fields while the one on the right displays a non-dimensional mode map of the flow fields.

The granular medium is energized by inputting energy via rotation. This rotational energy goes into several mechanisms, including translational, rotational, potential, acoustic, friction, and wear. The translational velocity induced by a rotary tumbler has been well characterized utilizing a variety of techniques such as PIV, MRI (Nitin Jain; 2002) (Florent Pignatel; 2012) and simulation (Pengfei Chen; 2008) (Valle, Romero; 2012). However, the rotation of the medium during the agitation has been characterized to a lesser degree.

The most common mode of operating a mass finishing device is somewhere between rolling and cascading. Rolling flows have been investigated and found to exhibit boundary layer like flow, with the highest translational velocities at the surface and along the internal wall boundary. 
Another important phenomenon, when investigating granular flows, is the existence of force chains. Force chains are the mechanisms by which boundary conditions communicate loads through the grain bed. Since not all grains participate in the force chain network, heterogeneous loading exists in the grain bed. This can result in some grains experiencing high loading and wear in specific regions of the flow. The goal was then to both repeat studies done by past researchers, who focused work on the linear motion of granular materials, and augment it with the inclusion of method and insight to measure rotational motion.

\section{Procedure}

Experimental Setup. After investigation and literary search, our work focused on the behavior of granular flow within a cylindrical rotary tumbler used in mass finishing applications. A tumbler design was then fabricated creating a clear tumbler with an interior diameter of $13.97 \mathrm{~cm}$ and inclusion of clear walls for viewing on all axes, stepper motor for variable speed control, and variable depth piston cylinder for $2 \mathrm{D}$ and $3 \mathrm{D}$ experimental analysis. The granular media, constrained to a single plane of motion, consisted of speckled glass spheres, each with a density of $2.23 \mathrm{~g} / \mathrm{cc}$ and a diameter of $1.5 \mathrm{~cm}$. Wheels supporting the tumbler were soft rubber and rotated freely on cylindrical roller bearings for a smooth controlled orbit.

Within experiments, marble number and drum rotation rate were variable with other conditions kept constant to study the various characteristic flows. A Photron camera, shooting at a speed of 500 frames per second and a resolution of 1024x 1024p, captured high-resolution data while not losing track of the particles. In order to light the media without glare, the room was kept dark while a diffuse studio light was used in coordination with a back-light to provide controlled lighting. After setting motor speed and waiting for a steady state, the Photron recorded up to four consecutive seconds of data (enough for most particles to travel at least once around the outer surface of the tumbler), which were collected in the form of raw images to be processed.

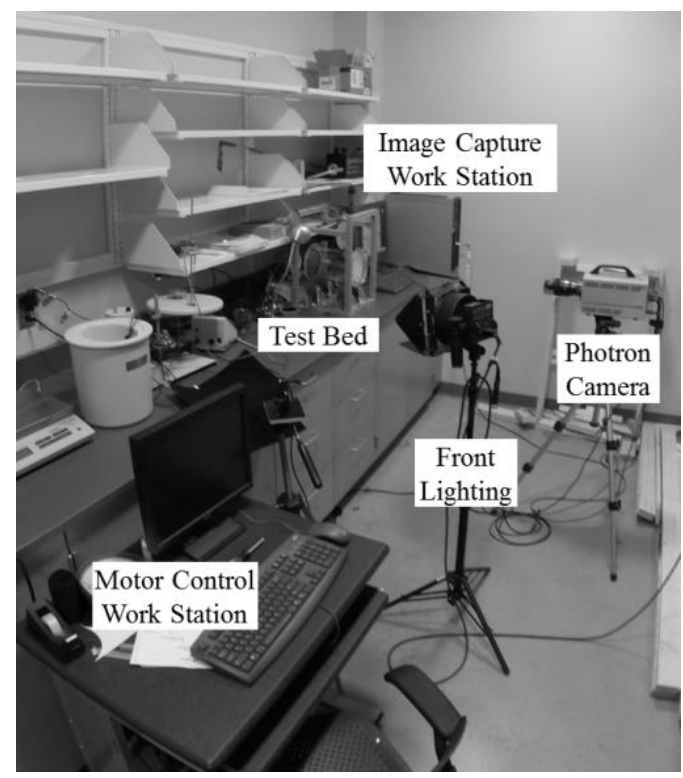

Figure 2: Experimental Study Setup

Methods. Most early studies qualify granular flow from experimental set-ups like the one in this report utilizing PIV or Particle Image Velocimetry to both qualify and quantify granular material motion. From this method, familiar looking images like the one shown below (in Figure 3-2) use 
vector plots superimposed over images to visually describe the flow while also providing data to be analyzed. PIV begins with a set of images with traceable particles, which all move within a relatively uniform fluid motion and do not radically change in qualities, such as shape, size and color, between frames. PIV functions through the utilization of interrogation windows. Between frames, like the ones shown in (Figure 3-1) below, images are correlated by transposing the integration window vertically and laterally to find probable path and speed resulting in a velocity vector for the given window. By keeping all particles within similarly sized interrogation windows and preventing particles from traversing into or out of a single window in a given time step, sets of image data can be used to form high resolution gradient fields.

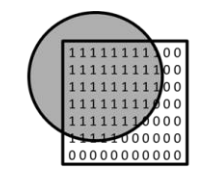

Frame 1

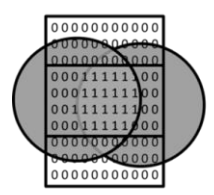

$(0,+2)=27$
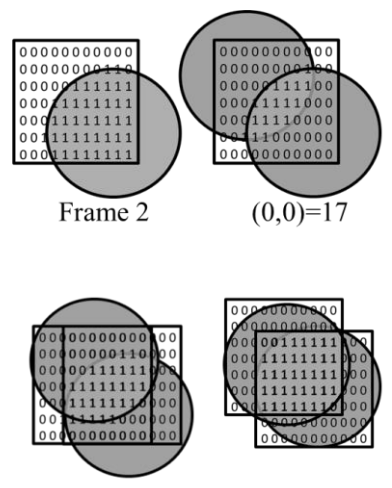

$(-2,0)=29$

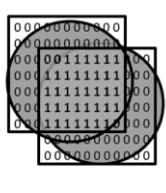

$(-2,+2)=37$
Overall Trend

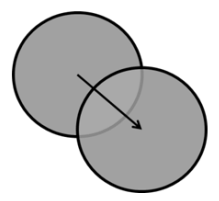

Figures 3-1 and 3-2: The images on the left show the before and after photos of a particle moving through the interrogation window. As images are translated, the binary addition of their pixels will result in either increasing or decreasing sum values. After comparing correlation values from various transpositions probable path emerges. The

image on the far right shows a processed result of PIV with arrows signifying general velocity trend at a point.

While our studies began with an initial foray into PIV, we ultimately used an algorithm built upon its architecture called PTV, or Particle Tracking Velocimetry, to begin characterization. While there are a multitude of PTV software packages available, this study builds off PTVlab (Brevis W; 2011), a Matlab add-on to PIVlab (the process outlined in Figure 4). While PIV is an excellent tool for measuring gradient flow fields, it did not allow for the tracking of media. While PIV can be used to ensemble average a flow, Particle Tracking Velocimetry (PTV) gives a tracking identity to every particle within a space and can give properties of an individual particle through space at any given time in addition to all of the PIV benefits mentioned above. PTV accomplishes this is by correlating each of the particles in addition to each of the frames. 


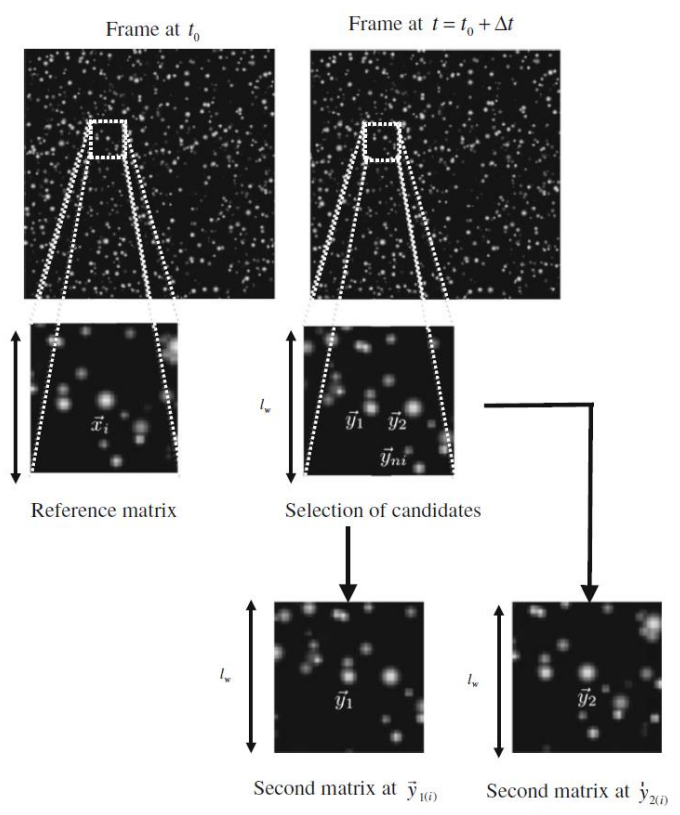

Figure 4: A description of the algorithm used by PTVlab which functions via relaxation and cross correlation.

This correlation takes place in two steps: cross correlation and relaxation. Relaxation uses a velocity data taken from a particle's last known trajectory to make an educated guess, assuming minimal acceleration, as to a particle's new location given a constant velocity and path. Cross Correlation finds neighbors that have similar properties (shape, size and color) to match a particle's identity between frames.

The final tool used was an adaptation of a preexisting method, often used in the medical field to adjust for organ movement when comparing MRI scans. Image registering, which works in a manner similar to both PIV and PTV, utilizes the values of pixels to determine when images match. Each pixel in a matrix was represented by a number, 0-255 (black to white). Two images are then converted into this numeric format before one is rotated with appropriate scaling and precautions. This prevents a loss of information as square matrixes are rotated and made to fit within the same bounds. If images were known to be a perfect match, the maximum value is obtained by multiplying each overlaying photo-matrix value.
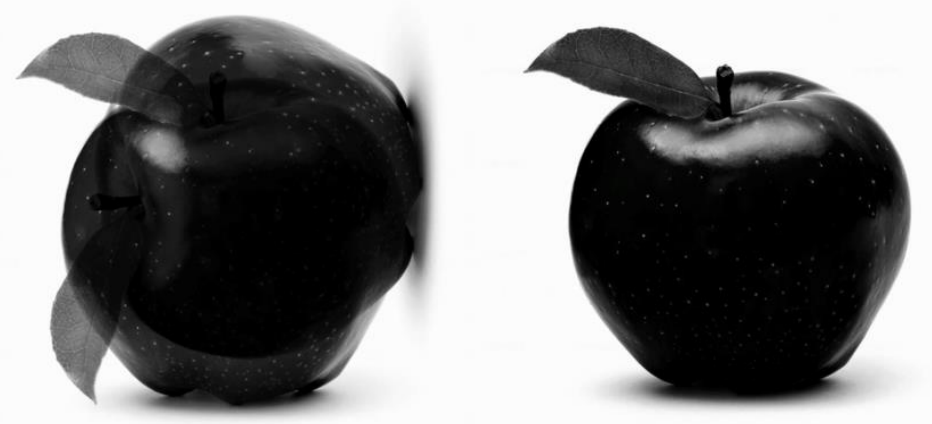

Figure 5: On the left an identical apple is superimposed over an identical apple mismatched by 90 degrees, and on the right the two images are matched to give a visual representation of a registered image. 
Data Processing. Images taken from the Photron camera were processed through a four-step method shown in Figure 6. Images were first fed through a built-in circle finding algorithm in Matlab which could locate and find particles of particular size before exporting an image with clear particle locations. From this point, the prepared images were processed in PTVlab to identify and track each of the particles in the flow field. In order to minimize misidentification of particles, the camera frame-rate was increased to ensure any given particle moved no more than one-half of its diameter per frame. Data from this process gave every particle's path and translational velocity at each point. Once each particle was identified, the image was cropped using a circular mask, which only leaves a given particle; this process was repeated for all particles. An example is presented in Figure 7. In order to estimate a particle's rotation, the second image, Figure 7-2 for example, is digitally rotated a known increment and registered with the first un-rotated image, Figure 7-1. For any two successive images, this process is repeated over angles: $-8^{\circ}<\theta<8^{\circ}$. The rotation with the maximum cross-correlation is assigned as the rotation for that grain over the interval of time between images. While this process ignored rotation in the $\mathrm{x}$ - and $\mathrm{z}$-directions, this issue will be addressed in future work.
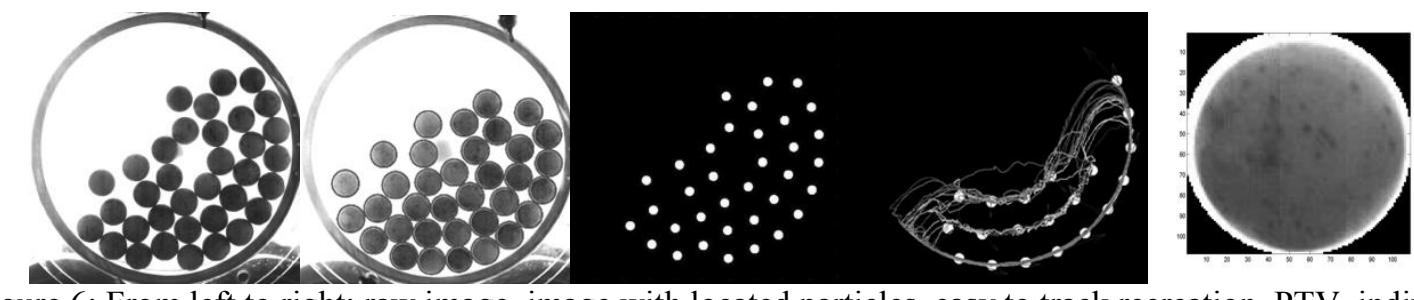

Figure 6: From left to right: raw image, image with located particles, easy to track recreation, PTV, individual masked for registering.

Unlike the stock apple image shown in the methods section, images in this study were not perfect matches-being before and after shots of the same particle. Therefore, while perfect correlation was not expected, peak correlation meant that a significant amount of particle features correlated.
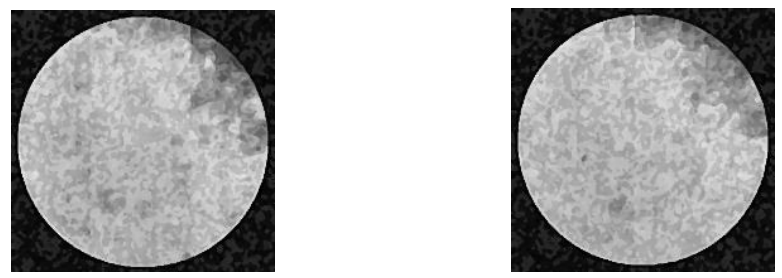

Figures 7-1 and 7-2: This piece of media has a countercolockwise rotation which took place in the timespan of 5 frames. Images collected during rolling (15\% Fill - Froude \#0.14).

From this data-providing particle identity, velocity, and rotation results of previous studies were duplicated and further results were compiled.

\section{Results}

Important Constants. To simulate and allow for the repeatability of research, the following table of constants showcases the various constants and properties possessed within experiments. Care was take in experimentation to remove media which possessed certain characteristics, such as size, which fell as an outlier amongst other media to preserve relative uniformity in experiments. 


\begin{tabular}{cccccc}
\hline Parameter (Marb/Marb) * & Value & Parameter (Marb/Drum) * & Value & Other Parameters & Value \\
\hline Young's Modulus & $64 \mathrm{GPa}$ & Young's Modulus & $2.96 \mathrm{GPa}$ & Marble Density & 2.23 g/cc \\
Poisson's Ratio & 0.2 & Poisson Ratio & 0.43 & Marble Diameter & $15.875 \mathrm{~mm}$ \\
Coefficient of Restitution & 0.97 & Coefficient of Restitution & 0.73 & Internal Drum Diameter & $13.97 \mathrm{~cm}$ \\
Coefficient of Friction & 0.9 & Coefficient of Friction & 0.43 & *Marb for Marble \\
\hline
\end{tabular}

Characteristic Modes. Of the six commonly recorded flows, findings in this study clearly recognized five. The most difficult modes to distinguish between were that of cascading and cataracting, made difficult due to the relative diameter of aggregate with respect to drum diameter. Slipping was defined by a low angle of repose. Slumping, like slipping, was defined by a point when aggregate "clings" to the rotating surface for a moment, before slipping back down the side in an oscillatory fashion. Rolling occurred when particles began to slide over the exposed upper surface aggregate. Cataracting is defined when the mass of particles begin to separate from the main flow. Finally, centrifuging occurred during complete loss of tumbling behavior as particles were flung outward and remained on the edge of the rotary tumbler.
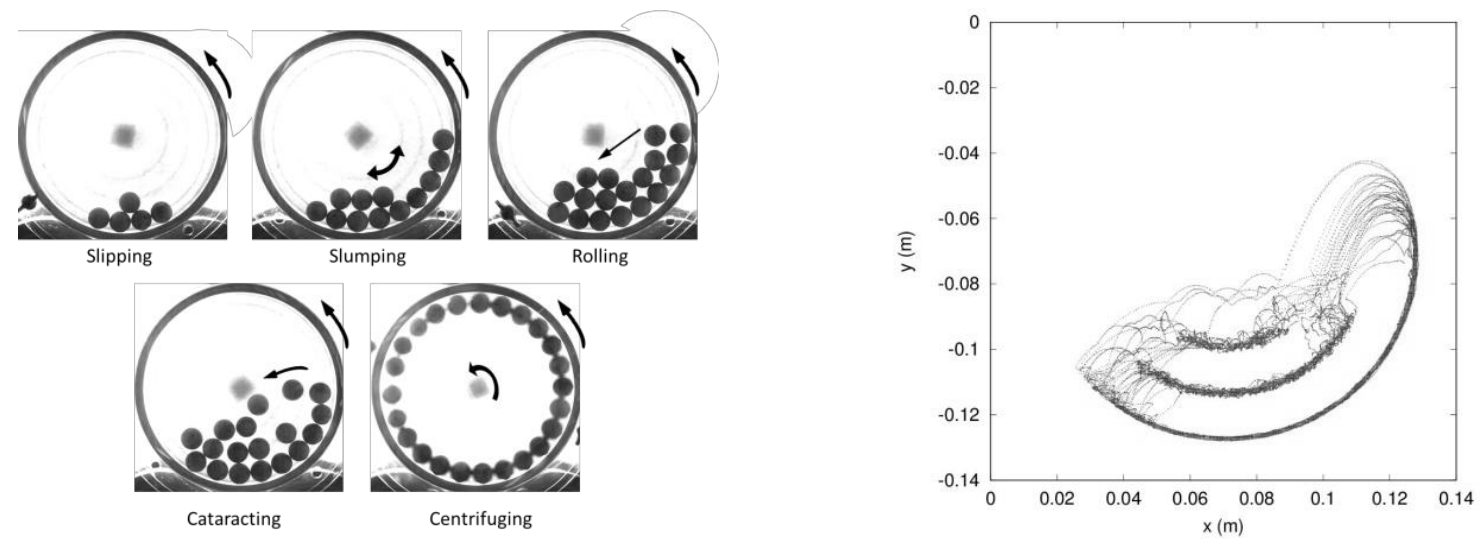

Figures 8-1 and 8-2: Images were collected for the following modes: Slipping (4\% Fill - Froude \#0.56), Slumping

(10\% Fill - Froude \#0.09), Rolling (15\% Fill - Froude \#0.14), Cataracting (15\% Fill - Froude \#1.44) and Centrifuging (20\% Fill - Froude \#). In the second figure, Cataracting particles are plotted with respect to position.

Figure 8-2 illustrates the formation of two boundary layers, one at the top surface and one toward the bottom. Grains in contact with the drum are imparted with velocity due to frictional drag, which is dictated by the friction and weight of the grain bed. The slump angle is dictated by how much kinetic energy the grains in the drum accumulate. As grains cascade down the free surface and meet the drum they are swept under the grain bed by the rotating drum. This is in contrast to grains near the center of the grain bed, which are near motionless. Thus, some grains continue to circulate while others remain nearly motionless toward the center of the grain bed.

Total Energy. Within a given system, the identification of the movement and transformation of energy within mass finishing devices remains a unified goal, yielding critical insight into its overall efficiency. The most common form of energy associated within a rotary tumbler is translational energy, but rotational energy plays a consistent role as media not only moves through the flow, but has a tendency to tumble while doing so. 


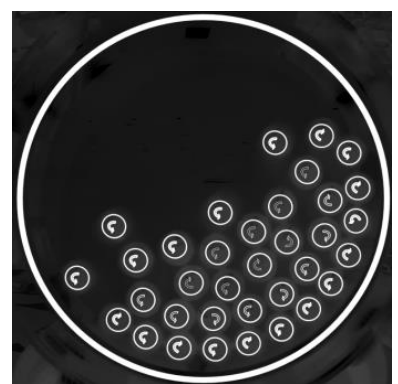

Figure 9: The image above is an artistic rendition of rotational force chains as generated within a rotary drum spinning counterclockwise; each particle affecting an opposite rotational force upon its neighbors.

To begin, plots of the characteristic depth of a particle, as a function of non-dimensional transverse, stream-wise, and rotational velocities, were generated. Transverse refers to xcomponent of particle velocity, while stream-wise is the y-component. Rotational nondimensional numbers are the ratio of a particle's rotation rate with respect to the drum's rotation rate. Within the non-dimensional plots, it can be seen that slipping and slumping are characterized by particles that do not change their bed depth over time, being fixed to a location, staying in set locations within the bed depth. It is hard to tell whether the drum was rotating in the clockwise or counter clockwise direction. In the stream-wise velocity graphs of rolling and cataracting, as media travels away from the drum wall, particles begin to travel with a clear velocity bias, betraying the counterclockwise rotation of our drum as particles rush across the exposed upper surface towards the point of lowest potential. Transverse velocity graphs show that, as particles reach the surface, they tend to accelerate as they are pushed from the main flow and, in the cases of cataracting, crash back onto the exposed surface. Finally, the centrifuging or phase space plots show the effects of gravity and drum shape as particles begin to accelerate or decelerate depending on their location within the flow.

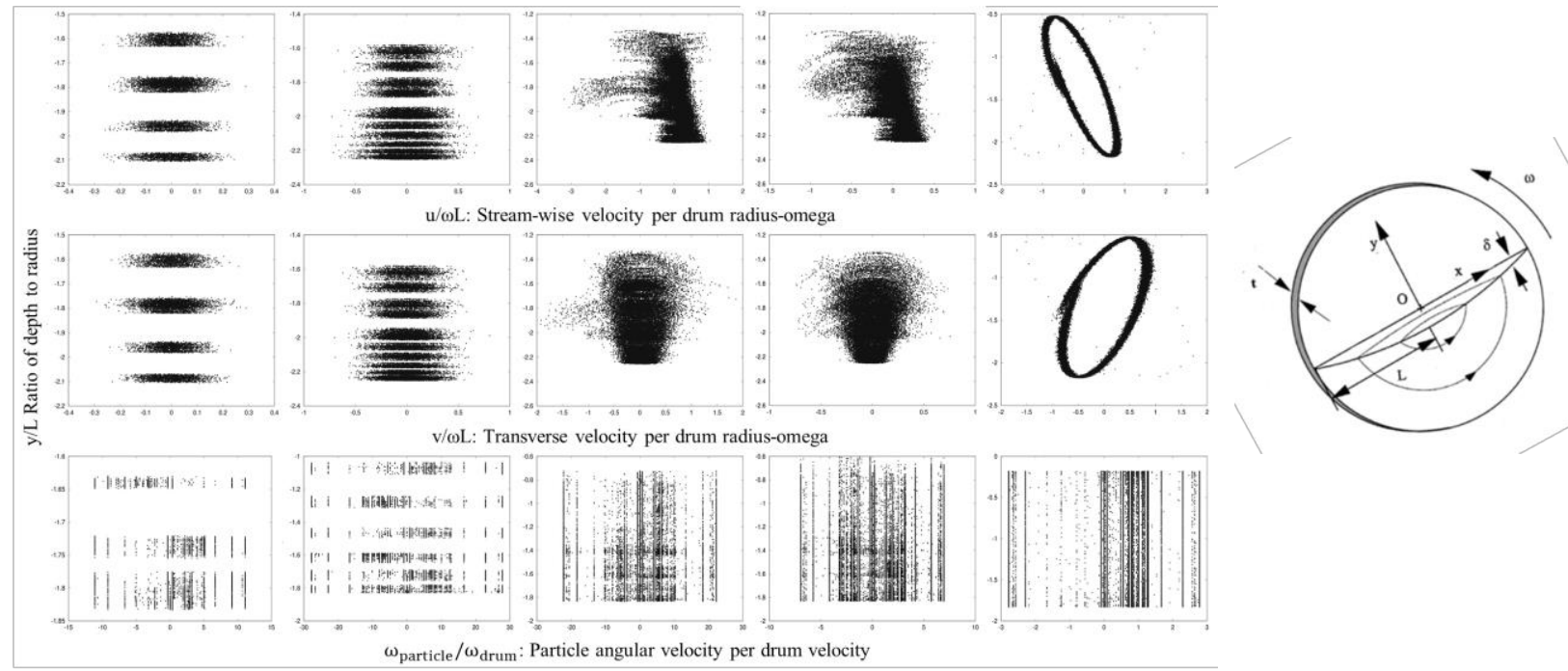

Figure 10: Plot of all particles: depth to radius as a function of stream-wise (top) and transverse (center) velocity per radius-omega, and particle angular velocity (bottom) per drum velocity at the conditions noted in Figure 8. Plots of non-dimensional translational velocities matched trends observed in a larger tumbler (Nitin Jain; 2002).

Histograms show the occurrences of certain energy, rotational or transverse, as well as give an indication of which type of energy contributes to the description of a certain system. As shown below, it follows that slipping, marked by particle spinning in place, would have high rotational 
energy and low translational energy, while cataracting, in which particles are flung onto the sides of the tumbler wall, would have high translational energy and little to no rotational energy.
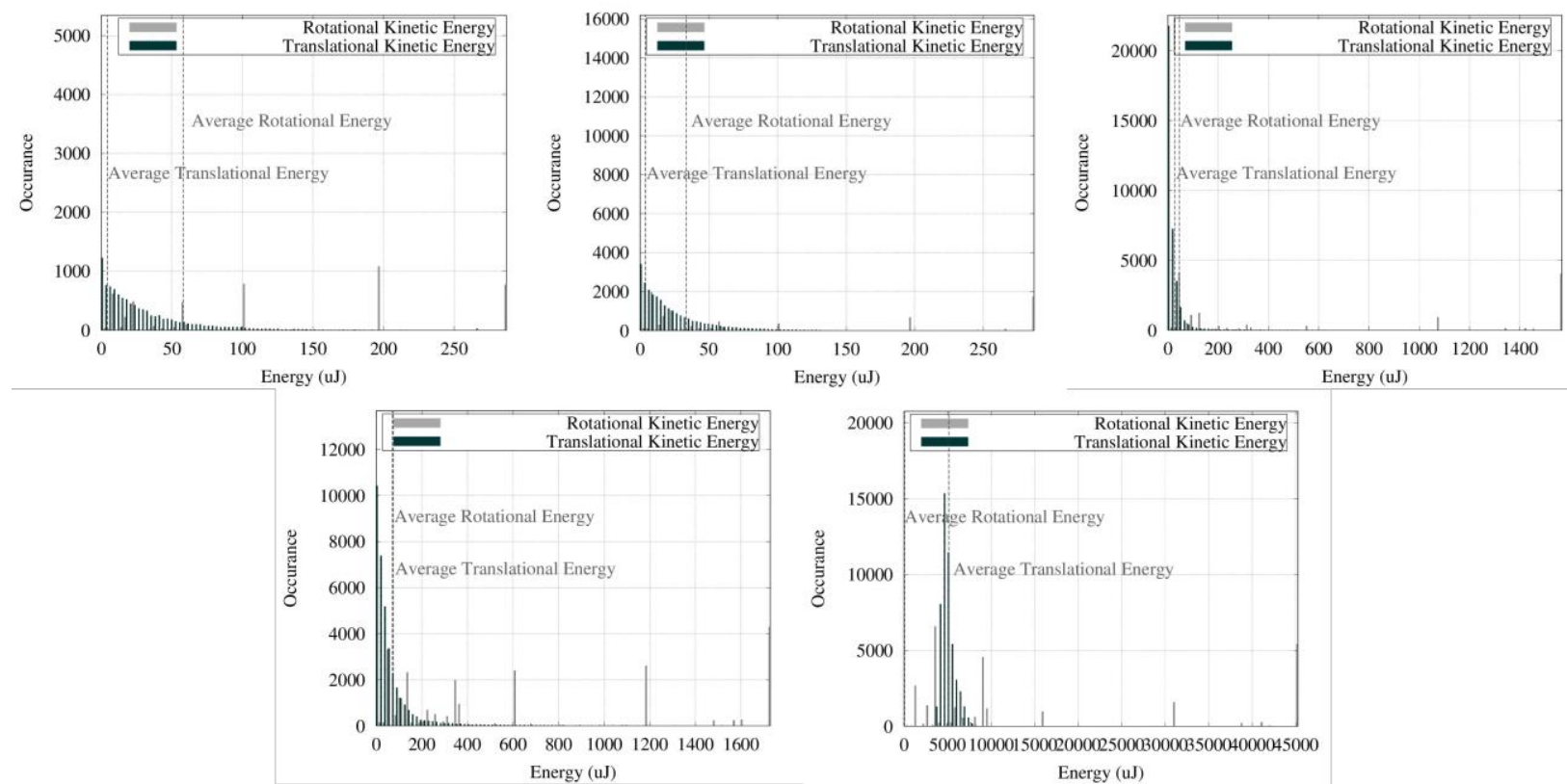

Figure 11: Histograms of translational and rotational energy occurrences at characteristic modes noted in Figure 8.

Finally, total energy plots (Figure 12) took a single system of 18 marbles with a percentage fill and varied the drum speed, which resulted in variable Froude numbers. From these data sets, rotational and translational energies were summed and plotted against the input Froude number. From this, energy could clearly be seen to increase as a function of drum speed, as expected. Dividing these results each by total energy, rotational energy can be seen contributing the majority of energy at lower energy states, while translational energy begins to play a more important role once rolling commences. Between rolling and centrifuging, both energy types vie for dominance over the system with clear evidence that optimization of granular flow is a plausible study for industry.
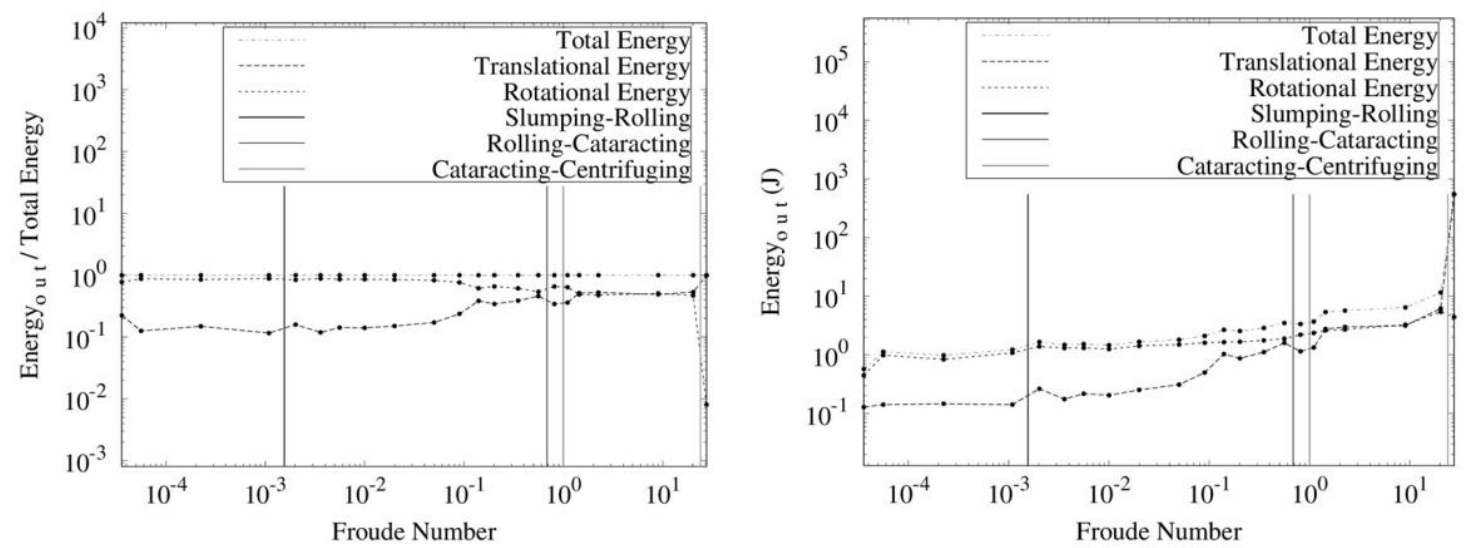

Figure 12: The plot on right shows energy over four seconds of time at a constant $15 \%$ fill as a function of Froude number while the one on the left charts translational and rotational energy over total measured energy as a function of Froude number. 


\section{Conclusions}

Results of previous studies closely corresponded to past insights. Using methods derived from those in the past, in coordination with available equipment, a method was created to measure rotation of individual particles within a two-dimensional granular flow. Bro-chains were an exciting scientific phenomenon that added to the existing idea of force chains. Force chains, in the statics sense, should take place normal to the points of contact on neighboring particles. In dynamics, knowledge of friction between particles makes it clear that forces will also occur tangential to a particle's surface resulting in rotational influences in particle interactions, i.e. brochains. In industry, the grinding and polishing of media is an incomplete science. Knowledge of particle interaction was used to machine items. However, aside from general recommendations of fill and speed, the understanding seemed incomplete. Processes that are not fully optimized imply room to decrease media consumption, increase effectiveness and decrease costs.

Overall, it was noted that the study of granular material motion worked well in the context of a companion study, demonstrating that by using a mathematical model for a physical system, one could save time and money by predicting and informing design. Similary, the characterization of the physical model informed simulations by advising algorithmic adjustment. While having access to a local computer cluster to help to speed calculations, these computations still took the vast majority of experimental and simulation time. To keep data collection and processing efficient, one should use minimal GUI interaction and run in parallel for efficient use of all cores.

Future areas of work into granular materials for the benefit of science include: the tracking of all individuals particles within a 3D environment via 3 Axis Gyroscopes or augmenting tracer particles within a three-dimensional flow field to glean rotation in all three axes. In contribution to industry, it has been noted that the processes, which remove the chips and flash off commercial products, represent a secondary application of the knowledge gleaned. Goals in this case would be to maximize the energy used to process materials, while decreasing the amount of media utilized by manipulating fill, media type and speed. It is within these ideas, a few of many, that research completed in this study could be applied to a next stage.

\section{References}

- Bendicks, C. "Improved 3-D Particle Tracking Velocimetry with Colored Particles." Journal of Signal and Information Processing 02.02 pp 59-71, (2011)

- Brevis W, Niño Y and Jirka GH. "Integrating cross-correlation and relaxation algorithms for particle tracking velocimetry.” Experiments in Fluids, 50 (1), pp 135-147. ISSN 0723-4864. (2011)

- Florent Pignatel, Caroline Asselin, Lucas Krieger, Ivan C. Christov, Julio M. Ottino, and Richard M. Lueptow. "Parameters and scalings for dry and immersed granular flowing layers in rotating tumblers" PHYSICAL REVIEW E 86, 011304. (2012)

- Homeniuk, Darren LN, David S. Nobes, and David J. Wilson. "Three-Dimensional Particle Tracking Velocimetry." The Canadian Society for mechanical Engineering. University of Alberta. T6G 2G8 (2010)

- Nitin Jain - J. M.Ottino - R. M.Lueptow. "An Experimental Study of the Flowing Granular Layer in a Rotating Tumbler." Physics of Fluids 14.2 (2002)

- Pengfei Chen, Julio M. Ottino, Richard M. Lueptow. "Subsurface granular flow in rotating tumblers: A detailed computational study." PHYSICAL REVIEW E 78, 021303 (2008)

- T. Deng, M.S. Bingley and MSA Bradley. "The influence of particle rotation on solid particle erosion rate of metals." Wear 256, pp 1037-1049, (2004)

- Valle, Romero. "Numerical Modeling of Granular Flows in Rotary Kilns." Thesis. Delft University of Technology. Literature Survey. (2012)

- Warr, S., Jacques, G. T. H. \& Huntley, J. M. "Tracking the translational and rotational motion of granular particles: use of high-speed photography and image processing." Powder Technol. 81, pp 41-56, (1994) 